\title{
Quantum steering in an asymmetric chain of nonlinear oscillators
}

\author{
Joanna K. Kalaga, ${ }^{1}$ Wiesław Leoński, ${ }^{* 1}$ Radosław Szczęśniak ${ }^{2}$ \\ ${ }^{1}$ Institute of Physics, University of Zielona Góra, Prof. Z. Szafrana 4a, 65-516 Zielona Góra, \\ ${ }^{2}$ Institute of Physics, Częstochowa University of Technology, Armii Krajowej 19, 42-200 Częstochowa
}

Received August 21, 2017; accepted September 27, 2017; published September 30, 2017

\begin{abstract}
We discuss here the possibility of generating steerable states in asymmetric chains comprising three Kerr-like nonlinear oscillators. We show that steering between modes can be generated in the system and it strongly depends on the asymmetry of internal couplings in our model. It can lead to the appearance of new steering effects, which were not present in symmetric models already studied in the literature.
\end{abstract}

Nonlocality is still one of the most intriguing phenomena which can occur in quantum-mechanical systems. In this paper, we concentrate on one of the classes of nonlocality - quantum steering. Although the first papers describing the steering were published by Schrödinger in 1935 [1], articles concerning the parameters which could be applied as steering criteria have appeared much later [2-4]. The relationships between steering and various types of quantum correlations such as quantum entanglement have also been intensively investigated [5-7]. Recently, the issues concerning systems and conditions for which we can obtain the strongest steering effect have become one of the crucial topics in studies of steering phenomena. It should be emphasized that the problems related to the steering and its relations with EPR paradox are of general nature, and were analyzed in various physical systems [8$10]$.

In this paper, we analyze the model involving a chain of three Kerr-like nonlinear oscillators, labeled by 1, 2, 3 and described by nonlinearity constant $\chi$, see Fig. 1 . The oscillators are mutually coupled by linear interactions which are characterized by the coupling parameters $\varepsilon_{12}$ end $\varepsilon_{23}$. Our system can be described by the following effective Hamiltonian:

$$
\hat{H}=\sum_{i=1}^{3}\left(\frac{\chi}{2} \hat{a}_{i}^{\dagger^{2}} \hat{a}_{i}^{2}\right)+\varepsilon_{12}\left(\hat{a}_{1}^{\dagger} \hat{a}_{2}+\hat{a}_{2}^{\dagger} \hat{a}_{1}\right)+\varepsilon_{23}\left(\hat{a}_{2}^{\dagger} \hat{a}_{3}+\hat{a}_{3}^{\dagger} \hat{a}_{2}\right),
$$

where the first term of the Hamiltonian describes the free evolution of three oscillators, whereas the other two represent the interaction between two oscillators (1-2 and 2-3). The operators $\hat{a}_{i}$, $\hat{a}_{i}^{\dagger}$ are boson annihilation and

\footnotetext{
*E-mail:w.leonski@if.uz.zgora.pl
}

creation operators, respectively, related to the mode $i$. Here, we assume that coupling constants are real, i.e. $\varepsilon_{i j}=\varepsilon_{i j}^{*}$.

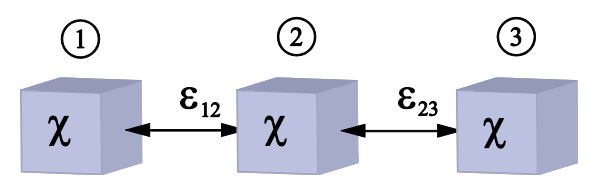

Fig. 1. The model. Three quantum nonlinear oscillators characterized by the same nonlinearity constant $\chi$ interact in pairs. The strengths of such interactions are determined by the parameters $\varepsilon_{12}$ and $\varepsilon_{23}$.

The model of nonlinear oscillators which we consider here is general enough to be applied in various physical situations and is usually studied with the use of both: numerical [11] and analytical [12] methods. For instance, such studies can be applied in quantum chaos [13-16], optical lattices [17-18], spin chains [11-12,19], superconducting systems [20-23] or QED systems [24] studies.

Our work is an extension of that presented in [25], where a fully symmetric system was considered. Here, we concentrate on the influence of internal couplings asymmetry on generation possibility of various steering effects. By the asymmetric chain, we mean that $\varepsilon_{12} \neq \varepsilon_{23}$. Additionally, we assume that coupling parameters are weak if compared to the values of nonlinearity parameters. To check the influence of the asymmetry, we will assume here that $\varepsilon_{12}$ remains constant $\left(\varepsilon_{12}=0.001 \chi\right)$, whereas the parameter $\varepsilon_{23}$ will vary from 0 to $6 \varepsilon_{12}$.

For the situation discussed here, the first oscillator initially is in the one-photon state, and the other subsystems are in the vacuum states. In consequence, as the system energy is conserved, its evolution remains closed within the set of three states (|001>, |010>, |100>) the system can be treated as a three qubit system.

As a measure of two-mode steering, we apply the parameter which is based on the inequality derived by Cavalcanti et al. [26]

$$
S_{i j}=\left\langle\hat{a}_{i} \hat{a}_{j}^{\dagger}\right\rangle\left\langle\hat{a}_{i}^{\dagger} \hat{a}_{j}\right\rangle-\left\langle\hat{a}_{i}^{\dagger} \hat{a}_{i}\left(\hat{a}_{j}^{\dagger} \hat{a}_{j}+\frac{1}{2}\right)\right\rangle,
$$


where $i, j$ label the system's modes. When the steering parameter $S_{i j}$ takes positive values, the mode $\mathrm{j}$ steers the mode i. From another side, when $S_{j i}$ is positive then steering in the opposite direction is present. Moreover, if both parameters $S_{i j}$ and $S_{j i}$ are greater than zero at the same time, symmetric steering appears - for the cases when only one of such two parameters, $S_{i j}$ or $S_{j i}$, is positive, the steering is asymmetric.

To analyze the steering effects appearing in our system, we have found the following analytical formulas defining steering parameters for all possible pairs of subsystems present in the model:

$$
\begin{aligned}
& S_{12}=-\frac{\left(\varepsilon_{12}^{2} \cos [t \omega]+\varepsilon_{23}^{2}\right)^{2}\left(\varepsilon_{12}^{2} \cos [2 t \omega]+\varepsilon_{23}^{2}\right)}{2 \omega^{6}} \\
& S_{21}=\frac{\varepsilon_{12}^{2} \sin ^{2}[t \omega]\left(\varepsilon_{23}^{2}\left(4 \varepsilon_{12}^{2} \cos [t \omega]-2 \varepsilon_{12}^{2}+\varepsilon_{23}^{2}\right)+\varepsilon_{12}^{4} \cos [2 t \omega]\right)}{2 \omega^{6}} \\
& S_{23}=-\frac{\varepsilon_{12}^{2} \sin ^{2}[t \omega]\left(\varepsilon_{12}^{4}-\varepsilon_{12}^{2} \varepsilon_{23}^{2}(\cos [2 t \omega]-4 \cos [t \omega]+1)+\varepsilon_{23}^{4}\right)}{2 \omega^{6}} \\
& S_{32}=-\frac{2 \varepsilon_{12}^{2} \varepsilon_{23}^{2} \sin ^{4}\left[\frac{1}{2} t \omega\right]\left(\varepsilon_{12}^{2} \cos [2 t \omega]+\varepsilon_{23}^{2}\right)}{\omega^{6}} \\
& S_{13}=\frac{\left(\varepsilon_{12}^{2} \cos [t \omega]+\varepsilon_{23}^{2}\right)^{2}\left(\varepsilon_{12}^{4}-\varepsilon_{12}^{2} \varepsilon_{23}^{2}(\cos [2 t \omega]-4 \cos [t \omega]+1)+\varepsilon_{23}^{4}\right)}{-2 \omega^{8}} \\
& S_{31}=\frac{2 \varepsilon_{12}^{2} \varepsilon_{23}^{2} \sin ^{4}\left[\frac{1}{2} t \omega\right]\left(\varepsilon_{23}^{2}\left(4 \varepsilon_{12}^{2} \cos [t \omega]-2 \varepsilon_{12}^{2}+\varepsilon_{23}^{2}\right)+\varepsilon_{12}^{4} \cos [2 t \omega]\right)}{\omega^{8}}
\end{aligned}
$$

where the frequency $\omega$ is defined by:

$$
\omega=\sqrt{\varepsilon_{12}^{2}+\varepsilon_{23}^{2}} .
$$

We can see that the steering parameters change periodically and the frequency $\omega$ depends on both coupling parameters $\varepsilon_{12}$ and $\varepsilon_{23}$. The ratio between them influences the values of the steering parameters and thus, the appearance of steering effects.

Figure 2 depicts the time-evolution of the parameters $S_{i j}$ for various values of the ratio $\varepsilon_{23} / \varepsilon_{12}$. The areas denoted by the white color correspond to the situations when $S_{i j} \leq 0$. That means that mode $\mathrm{j}$ does not steer mode i. The colored areas represent situations for which such steering appears. Here, we see that the appearance of the steering effects strongly depends on the ratio of the strengths of interactions between oscillators $\varepsilon_{23} / \varepsilon_{12}$.

For $\varepsilon_{23} / \varepsilon_{12}=1$ (symmetric chain) the mode 1 steers mode 2 and mode 3 steers mode 2. Additionally, two other steering effects are present $-1 \rightarrow 3$ and $3 \rightarrow 1(i \rightarrow j$ denotes the situation when mode $\mathrm{i}$ steers $\mathrm{j})$. Moreover, steering between two boundary modes ( 1 and 2 ) does not appear simultaneously in two directions, so the steering present there is asymmetric. Such behavior corresponds to the results obtained by Olsen for small (symmetric) BoseHubbard chains [25].

When $\varepsilon_{23} / \varepsilon_{12} \neq 1$ (asymmetric chain), steering is generated for other pairs of modes. If $\varepsilon_{23} / \varepsilon_{12}<0.42$ two non-zero steerabilities corresponding to the pairs $1 \rightarrow 2,2 \rightarrow 1$ and $1 \rightarrow 3,2 \rightarrow 3$ appear in the system. The first oscillator steers simultaneously two other oscillators and additionally, subsystem 2 steers at the same time subsystems 1 and 3 . Moreover, the same as for the symmetric chain, steering which appears in the system acts in one direction. When the strength of interaction between the modes 2 and 3 increases, and $1<\varepsilon_{23} / \varepsilon_{12}<2.42$, the second oscillator stops to steer two other subsystems. Further increase of the value of coupling parameter $\varepsilon_{23}$ leads to the disappearance of the steering $3 \rightarrow 1,3 \rightarrow 2$ (for $\varepsilon_{23} / \varepsilon_{12}>2.42$ ). In consequence, for such a case only steering from subsystem 1 remains there. Moreover, for $\varepsilon_{23} / \varepsilon_{12}>2.42$ the first oscillator steers two others almost permanently. What is interesting, when the ratio $0.42 \leq \varepsilon_{23} / \varepsilon_{12}<1.00$ all six steering parameters are positive, so all possible steerings are present in the system. It is one of the most relevant features appearing in our model which was not present in the symmetric system considered in [25].

In Figure 3 we show how the maximal values of $S_{i j}$ depend on the value of $\varepsilon_{23} / \varepsilon_{12}$. We see that even small changes in $\varepsilon_{23} / \varepsilon_{12}$ can cause noticeable variations in the maximal values of steering parameters. When $\varepsilon_{23} / \varepsilon_{12}$ is small, steering between oscillators 1 and 2 dominates. In fact, such a case corresponds to the situation when the last oscillator can be neglected and the system reduces to a qubit-qubit one. With an increasing value of $\varepsilon_{23} / \varepsilon_{12}$ the contribution of the steerabilities corresponding to the subsystems involving oscillator 3 become more pronounced, contrary to the steering between subsystems 1 and 2 (in the both directions), which is in decline. The strongest steering between modes 1 and 3 can be achieved for two values of $\varepsilon_{23} / \varepsilon_{12}$ (for each of the two steering directions). Thus, there are $\varepsilon_{23} / \varepsilon_{12}=\{0.27 ; 3.72\}$ for the steering $1 \rightarrow 3$ and $\varepsilon_{23} / \varepsilon_{12}=\{0.58 ; 1.73\}$ for $3 \rightarrow 1$.

When the value of coupling between modes 2 and 3 is much larger than that for 1 and 2, only $S_{21}$ and $S_{31}$ remain greater than zero. However with still increasing $\varepsilon_{23} / \varepsilon_{12}$ both steering parameters disappear asymptotically. Thus, we see that changes in the value of $\varepsilon_{23} / \varepsilon_{12}$ can lead to a variety of situations for which manifold steering effects can appear.

We have discussed here the short chain of three nonlinear oscillators. We assumed here that the system's evolution starts from the state for which only the first oscillator is in the one-photon state, whereas the two others are in the vacuum state. In this work, we have shown how the asymmetry in internal couplings can lead to the appearance of new steering effects. 

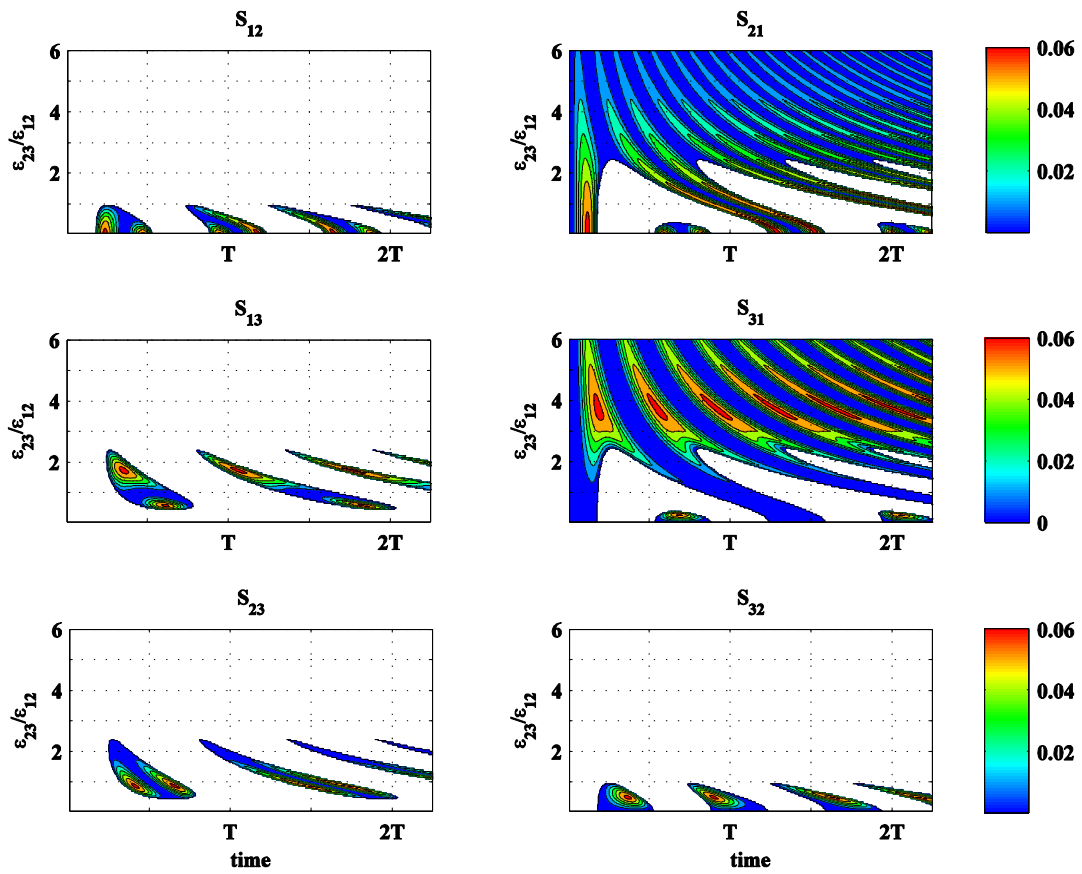

Fig. 2. Time-evolution of steering parameters $S_{i j}$. Time is measured in units of $\mathrm{T}$, where $\mathrm{T}=1 / \omega$ is a period of the evolution when $\varepsilon_{23} / \varepsilon_{12}=1$.

If we compare the results presented here to those which were discussed in [25], we see that the presence of such asymmetry can allow us to obtain a variety of new steering effects which were absent in the symmetric system. The main result presented here is that all possible six steerabilities can simultaneously appear in the system, contrary to the situation considered in [25], where only four of them could be present.

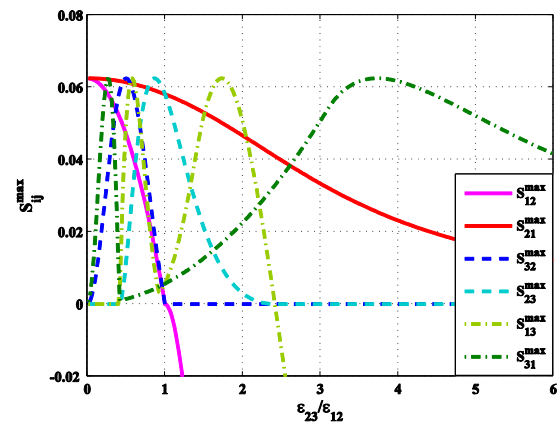

Fig. 3. The maximal values of $\mathrm{S}_{\mathrm{ij}}$ as a function of $\varepsilon_{23} / \varepsilon_{12}$.

\section{References}

[1] E. Schrödinger, Math. Proc. Camb. Phil. Soc. 31, 555 (1935).

[2] M.D. Reid, Phys. Rev. A 40, 913 (1989).

[3] E.G. Cavalcanti, M.D. Reid, J. Modern Opt. 54, 2373 (2007).

[4] S.P. Walborn, A. Salles, R.M. Gomes, F. Toscano, P.H. Souto Ribeiro, Phys. Rev. Lett. 106, 130402 (2011).

[5] H.M. Wiseman, S.J. Jones, A.C. Doherty, Phys. Rev. Lett. 98, 140402 (2007).
[6] S.J. Jones, H.M. Wiseman, A.C. Doherty, Phys. Rev. A 76, 052116 (2007).

[7] J.K. Kalaga, W. Leoński, Quant.Inf. Process 16, 175 (2017).

[8] Q. He, Z. Ficek, Phys. Rev. A 89, 022332 (2014).

[9] S. Kiesewetter, Q.Y. He, P.D. Drummond, M.D. Reid, Phys. Rev. A 90, 043805 (2014)

[10] K. Bartkiewicz, A. Cernoch, K. Lemr, A. Miranowicz, F. Nori, Scient. Reports 6, 38076 (2016).

[11] A. Barasiński, B. Brzostowski, R. Matysiak, P. Sobczak, D. Woźniak, In: R. Wyrzykowski, J. Dongarra, K. Karczewski, J. Wasniewski editor, Parallel Processing and Applied Mathematics (PPAM 2013), Lecture Notes in Computer Science, vol 8385. Springer, Berlin, Heidelberg (2014).

[12] A. Drzewiński, J. Sznajd, Physica A 170, 415 (1991).

[13] G.J. Milburn C.A. Holmes, Phys. Rev. A 44, 4704 (1991).

[14] W. Leoński, Physica A 233, 365 (1996).

[15] A. Kowalewska-Kudłaszyk, J.K. Kalaga, W. Leoński, Phys. Lett. A 373, 1334 (2009).

[16] J.K. Kalaga W. Leo ński, Proc. SPIE 10142, 101421L (2016).

[17] A. Barasiński, W. Leoński, T. Sowiński, J. Opt. Soc. Am. B 31, 1845 (2014).

[18] A. Barasiński, W. Leoński. Quant. Inf. Process 16, 6 (2017).

[19] D. Woźniak, A. Drzewiński, G. Kamieniarz, Acta Phys. Polon. A 130, 1395 (2016).

[20] R. Szczęśniak, D. Szczę́niak, E.A. Drzazga, Solid State Commun. 152, 2023 (2012).

[21] A. P. Durajski, R. Szczęśniak, M.W. Jarosik, Phase Trans. 85, 727 (2012).

[22] R. Szczęśniak and A. P. Durajski, Solid State Sciences 25, 45 (2013).

[23] X. Wang, A. Miranowicz, H.R. Li, F. Nori, Phys. Rev. A 94, 053858, (2016).

[24] Y.X. Liu, X.W. Xu, A. Miranowicz, F. Nori, Phys. Rev. A, 89, 043818 (2014).

[25] M.K. Olsen, Phys. Rev. A 92, 033627 (2015).

[26] E.G. Cavalcanti, Q.Y. He, M.D. Reid, H.M. Wiseman, Phys. Rev. A 84, 032115 (2011) 